\title{
Familias transnacionales, familias inmigrantes: Reflexiones sobre su inclusión en la escuela
}

\author{
Transnational Families, Immigrant Families:
}

Reflections on their Inclusion in School

\author{
María Fernández-Hawrylak y Davinia Heras Sevilla ${ }^{1}$
}

\section{Resumen}

El objetivo del presente artículo es describir el impacto que las migraciones tienen en las familias a través de una revisión bibliográfica sobre migración internacional como metodología de trabajo. Se examinaron artículos publicados entre 1998 y 2014 indexados en las bases de datos Web of Science y Scopus. Con el análisis de los artículos seleccionados se construyó un cuerpo teórico desde el cual se presentan los conceptos de familia transnacional, feminización de la migración mundial, concepción de la paternidad, fragmentación de la familia, reagrupación familiar, así como las principales dificultades de las familias migrantes en el país de destino que dan lugar a situaciones de exclusión de los padres y de las/los niñas/os en la escuela. Entre los resultados destaca que, aunque la separación geográfica pueda generar beneficios económicos, estas familias sufren una desestabilización que crea nuevas formas de funcionamiento para mantener sus vínculos y llevar a cabo el cuidado y la educación de los hijos desde la distancia. Como conclusiones se recogen reflexiones e investigaciones en torno a la inclusión de las/los niñas/os inmigrantes en el sistema educativo, y sobre la necesidad de un compromiso con sus familias para apoyarlas en el proceso educativo.

\section{Palabras clave}

Migración, transnacionalismo, familia transnacional, familia inmigrante, inclusión, escuela.

\section{Abstract}

In this paper, we describe the impact of migration in the families through a bibliographic review on international migration as our work methodology. We examined the papers published between 1998 and 2014 that are indexed in Web of Science and Scopus databases. A theoretical body was constructed from the analysis of the selected articles, from which we present the concepts of transnational family, feminization of global migration, conception of parenthood, family fragmentation, family regrouping, and the main difficulties of migrant families in the country of destination that lead to situations of exclusion of both parents and children in school. Although a geographical separation can generate economic benefits, these families suffer a destabilization that creates new ways of functioning in order to maintain their links and to carry out the care and education of the children from distance. As conclusions we collect some reflections and researches about the inclusion of immigrant children in the educational system, and about the need for a commitment with their families to support them in the educational process.

\section{Keywords}

Migration, transnationalism, transnational family, immigrant family, inclusion, school. 


\section{Introducción}

La relación entre los movimientos migratorios y la globalización es ampliamente conocida entre la comunidad científica. En la actualidad, son numerosos los ciudadanos de diferentes culturas y nacionalidades que emigran hacia otros países en busca de un futuro mejor para ellos y para sus familias. En general, se trata de flujos migratorios desde lugares no industrializados a destinos industrializados (migración laboral sur-norte y norte-norte). Sin embargo, debido a las políticas de extranjería de los países del norte, la cercanía cultural y lingüística entre los países del sur, y el coste que supone la migración, en los últimos años se ha producido un incremento en los movimientos migratorios interregionales (Mora, 2008), principalmente hacia el sur en países de América Latina (migración sur-sur). En cualquier caso, es innegable el impacto de la emigración en el contexto internacional, ya que transporta a distintos grupos humanos a lo largo de la geografía mundial. En este sentido, el Fondo de Población de las Naciones Unidas (UNFPA, 2013), estima que el número de ciudadanos que habitan fuera de su país de origen se ha incrementado de 155 millones en 1990 a 214 millones en 2010, lo que constituye el 3\% de la población mundial actual. La Organización Internacional del Trabajo (OIT, 2006) indica, además, que el 49\% de los migrantes en el mundo son mujeres, la mayoría de ellas en edad reproductiva.

Pero la relevancia del fenómeno migratorio surge no solo por datos de índole cuantitativa, sino por las características cualitativas de la inmigración: heterogeneidad de los colectivos (distintas nacionalidades, orígenes socioeconómicos y socioeducativos diversos), grupos de edad (jóvenes en edad de trabajar, niños y jóvenes en el sistema educativo) y el proyecto migratorio (familias transnacionales - familias inmigrantes) (Santos, 2008).

Por todo ello, resulta crucial analizar la repercusión de estos procesos migratorios en los sujetos protagonistas. En este sentido, la familia es el contexto de la vida cotidiana que más ha sido afectado por la migración como proceso, y que requiere un abordaje específico que permita explicaciones (Martín, 2007).

La migración como proceso genera un importante impacto (social, cultural, político y económico) tanto en el país de origen como en el país de destino, así como en el grupo migrante que hace grandes esfuerzos por mantener los vínculos familiares a través de las fronteras. De esta manera, la familia desempeña un papel fundamental en este proceso migratorio, puesto que se establecen y construyen lazos que superan la co-residencia y la presencia física. Todo ello supone reconfiguraciones y ajustes que obligan a estudiar a las familias no solo desde el espacio nacional, sino desde el contexto transnacional (Parella, y Cavalcanti, 2006; Zapata, 2009).

Los procesos migratorios actuales se han convertido en un proceso transformador con implicaciones familiares profundas. En este contexto migratorio internacional se produce un reacomodo de las relaciones de género y generacionales, que interviene en la negociación de los vínculos dentro del grupo doméstico e influye en las modalidades de reagrupaciones familiares y en las experiencias de los hijos de la familia migrante, tanto en los lugares de origen como en los de destino (Pedone, 2006).

Si bien es cierto que no toda inmigración provoca los mismos efectos sobre las familias, también lo es el que se producen consecuencias de distinto calado cuando media la distancia del país de origen o cuando se lleva a cabo la reintegración o reunificación en el país de llegada. Un importante porcentaje de familias inmigrantes sufren procesos de reorganización interna en cuanto a sus estructuras, relaciones y funciones, influyendo de manera trascendental en la educación de los hijos (Carrasco, Pàmies y Beltrán, 2009). La familia emerge, en cualquier caso, como espacio, tiempo y lugar donde se experimentan con 
mayor intensidad las relaciones humanas, la identidad y las redes que se crean en el proceso migratorio (Martín, 2007).

Este artículo, a través de la revisión sistemática de la literatura, pretende describir cómo los proyectos migratorios familiares impactan en las familias, donde en la mayoría de los casos se parte de una condición de familias transnacionales para convertirse en familias de inmigrantes, siendo las/los niñas/os quienes se ven más afectadas/os por esta condición en la que se buscan nuevas oportunidades y una mejora de la calidad de vida para todo el núcleo familiar, que suele incluir no solo a madres y padres e hijas/os, sino a abuelas/os, hermanas/os y primas/os entre otros.

Se realizó una revisión bibliográfica para elaborar un artículo que recopilase información relevante sobre las familias transnacionales y su inclusión el contexto escolar. La revisión descriptiva proporciona una puesta al día sobre conceptos útiles en áreas en constante evolución, como es el caso de la familia. Este tipo de revisiones interesa a muchas personas de campos conexos, porque leer buenas revisiones es la mejor forma de estar al día en nuestras esferas generales de interés, a la vez que son de gran utilidad para aproximarse a los procesos de enseñanza-aprendizaje (Day, 2005: 161).

\section{Metodología}

El objetivo principal de este trabajo es describir el impacto que las migraciones tienen en las familias a través de la revisión de la literatura sobre migración internacional. Para ello, se plantearon algunos interrogantes que permitieran explicar distintos aspectos sobre la problemática planteada: ¿qué son familias transnacionales?, ¿cómo se forman estas familias?, ¿cómo mantienen sus vínculos?, ¿cuáles son las dificultades y desafíos con los que se encuentran en la sociedad y en la escuela?, y ¿cómo se puede responder a sus necesidades?

La metodología utilizada es la revisión bibliográfica, que desde una perspectiva cualitativa es la que mejor responde al objetivo del trabajo. En educación, esta metodología pretende mejorar la calidad de los procesos educativos y ayudar a los educadores en su reflexión sobre la práctica educativa (Bisquerra, 2009), que es lo que nos mueve en este caso. Mientras que desde un enfoque cuantitativo se suele presentar una extensa revisión bibliográfica para describir el objeto de estudio y proporcionar un marco a las preguntas de investigación e hipótesis, desde una vertiente cualitativa la revisión de la literatura adopta una función más comprensiva y mucho menos prescriptiva (Rodríguez y Valldeoriola, 2009).

Se trata, por tanto, de un trabajo descriptivo donde se exploran aspectos como la feminización de la migración mundial, la concepción de la paternidad, la fragmentación de la familia, la reagrupación familiar, y otras dificultades a las que se enfrentan las familias que deciden emprender proyectos migratorios. Esto puede llevar a reflexionar sobre la inclusión de las/los niñas/os inmigrantes en nuestro sistema educativo sobre la base de un conocimiento mayor de lo que supone la migración en el sistema familiar.

La metodología propuesta se compone de cuatro fases: 1) definición del problema; 2) búsqueda de la información; 3) organización de la información; 4) análisis de la información. De este modo:

1. Definición del problema: se determinaron las preguntas anteriormente referidas.

2. Búsqueda de la información: para la localización de los documentos se realizó una revisión sistemática en las bases de datos bibliográficas Web of Science y Scopus de artículos publicados entre 1998 y 2014. Para llevar a cabo la búsqueda se utilizaron las palabras clave «transnacional $\square$ », «migración 
$\square$ », «familia $\square$ transnacional $\square$ », ampliándola mediante los comodines para obtener resultados más variados. Se registró una cantidad considerable de resultados tras la combinación de las diferentes palabras clave (tabla I), deteniendo la búsqueda al considerar que tal cantidad permitiría trazar un trabajo con un horizonte claro sobre el estado del conocimiento.

3. Organización de la información: se definieron criterios de inclusión y exclusión, y un conjunto de variables que permitieran analizar posteriormente los artículos localizados, seleccionando aquellos que arrojasen información sobre distintos aspectos relacionados con las familias transnacionales, considerando nuevas categorías o variables a medida que iban surgiendo durante el examen documental. Se tomaron como criterios de inclusión todos los documentos publicados en castellano que abordaran la formación y el funcionamiento de las familias transnacionales, y las dificultades para su integración e inclusión en el país de destino. Los restantes fueron descartados debido a que su información no respondía a las preguntas de investigación, eran redundantes y/o correspondían a otras áreas de conocimiento (literatura, medicina...). Para la selección se atendieron los siguientes criterios:

a) Estudios con diseños de investigación de naturaleza cualitativa y socio-critica afines a la problemática del estudio.

b) Trabajos donde se conceptualiza la familia transnacional y se aborda su formación.

c) Investigaciones sobre el impacto de la migración tanto en la unidad familiar como en cada uno de los sub-sistemas familiares.

d) Artículos sobre la integración/inclusión de las familias transnacionales-inmigrantes en la escuela.

Para el primer filtrado, se consideró la revisión del título y en algunos casos del resumen, lo cual permitió seleccionar 45 artículos. Tras haber reducido considerablemente la cantidad de resultados, los seleccionados se sometieron a una valoración de acuerdo a los criterios de inclusión y exclusión propuestos en este trabajo, disminuyendo su número hasta 23. La información procedente de los artículos seleccionados se completó con libros y capítulos de libros e informes.

4. Análisis de la información: finalmente se procedió a construir un cuerpo teórico centrado en los siguientes puntos: (1) conceptualización y formación de las familias transnacionales; (2) efectos de la migración en las familias y sus miembros; (3) dificultades de las familias transnacionales-inmigrantes en la escuela, y su consideración desde un planteamiento inclusivo e intercultural.

\section{Tabla I. Resultdos de las búsquedas efectuadas por término}

TÉRMINO DE BÚSQUEDA

\begin{tabular}{lllllll}
\hline & & Transnacional $\square^{1}$ & & Migración $\square^{2}$ & Familia $\square$ Trasnacional $\square^{3}$ \\
\hline Año & WoS & Scopus & WoS & Scopus & WoS & Scopus \\
\hline 1998 & 0 & 3 & 12 & 18 & 0 & 0 \\
\hline 1999 & 0 & 0 & 5 & 14 & 0 & 0 \\
\hline 2000 & 4 & 3 & 4 & 7 & 0 & 0 \\
\hline 2001 & 1 & 2 & 6 & 19 & 0 & 0
\end{tabular}


TÉRMINO DE BÚSQUEDA

\begin{tabular}{|c|c|c|c|c|c|c|}
\hline \multirow[b]{2}{*}{ Año } & \multicolumn{2}{|c|}{ Transnacional $\square^{\prime}$} & \multirow{2}{*}{\multicolumn{2}{|c|}{$\frac{\text { Migración } \square^{2}}{\text { Scopus }}$}} & \multicolumn{2}{|c|}{ Familia $\square$ Trasnacional $\square^{3}$} \\
\hline & WoS & Scopus & & & WoS & Scopus \\
\hline 2003 & 13 & 6 & 64 & 17 & 1 & 1 \\
\hline 2004 & 15 & 7 & 62 & 21 & 1 & 1 \\
\hline 2005 & 25 & 0 & 97 & 21 & 2 & 0 \\
\hline 2006 & 48 & 4 & 111 & 17 & 4 & 1 \\
\hline 2007 & 42 & 0 & 137 & 28 & 7 & 0 \\
\hline 2008 & 49 & 4 & 154 & 32 & 2 & 0 \\
\hline 2009 & 83 & 29 & 198 & 45 & 8 & 3 \\
\hline 2010 & 56 & 16 & 193 & 42 & 9 & 1 \\
\hline 2011 & 66 & 20 & 193 & 51 & 7 & 2 \\
\hline 2012 & 70 & 18 & 259 & 78 & 10 & 2 \\
\hline 2013 & 84 & 37 & 263 & 49 & 5 & 2 \\
\hline \multirow[t]{2}{*}{2014} & 124 & 31 & 334 & 72 & 9 & 0 \\
\hline & 692 & 182 & 2142 & 547 & 65 & 13 \\
\hline
\end{tabular}

1 Los resultados incluyen los términos transnacional, transnacionales, transnacionalismo...

2 Los resultados incluyen los términos migración y migraciones.

3 Los resultados incluyen los términos familia, familias, familiar, familiares, transnacional, transnacionales... No tienen por qué aparecer juntos en el texto, pero sí aparecen ambos.

\section{Resultados}

\section{1. La migración internacional: las familias transnacionales}

La familia transnacional se constituye a partir de los procesos migratorios. Esto permite evidenciar nuevas formas de relación y vínculos que se establecen entre los migrantes y su familia ubicada en el país de origen (Zapata, 2009). Los miembros que configuran este tipo de familias viven separados físicamente unos de otros, pero son capaces de mantener vínculos que les permiten sentirse integrantes de una unidad y percibir bienestar a pesar de la distancia, construyendo grupos familiares en los que, a pesar de la separación geográfica entre el migrante y su familia, las relaciones no se fracturan (Zapata, 2009; Mejía, y Arriaga, 2012; Cerda, 2014). Para denominar a este tipo de familias se han utilizado términos como multilocal, transcontinental, internacional o multisited (Parella, y Cavalcanti, 2006; Parella, 2007; Zapata, 2009).

Debido a la distancia, a la pluralidad de formas de contacto y al carácter esporádico de los encuentros físicos, las familias transnacionales deben construir y reconfigurar sus nociones de familia, sus vínculos emocionales y sus acuerdos económicos deliberadamente, puesto que la identidad familiar no surge sobre la base de la interacción cotidiana reducida al espacio territorial nacional (Martín, 2007). Estas familias inciden intencionalmente sobre los lazos familiares, y reducen los efectos de la distancia al forjarlos de manera permanente a través de múltiples vías de contacto simbólico y real (remesas), y redes transnacionales (redes migratorias).

En la formación de hogares transnacionales se utiliza el transnacionalismo como forma de hacer frente a las estrategias productivas y reproductivas. Cabe señalar que el concepto de familia transnacional es abordado en relación a los términos hogar transnacional, unidad doméstica transnacional, 
grupo doméstico transnacional, hogar multinuclear, etc., donde se producen las relaciones sociales y las prácticas de producción (aspectos económicos) y de reproducción (aspectos sociosimbólicos y culturales, formación de los valores y afectividad) que se dan en estas relaciones, así como la interrelación que de dichos aspectos se deriva (Gonzálvez, 2007; Rivas y Gonzálvez, 2011).

Salazar Parreñas (2001), citado por Oso (2008), distingue tres tipos de hogares transnacionales: (1) un progenitor fuera (padre o madre); (2) dos progenitores fuera; (3) un hijo adulto fuera. En general, estos hogares se componían de un varón migrante que sustentaba la economía familiar, y que obligadamente vivía separado de su esposa e hijos, quiénes permanecían en el país de origen. Sin embargo, los estudios sobre paternidad trasnacional son escasos, centrándose en los aspectos de carácter económico de la separación del padre y de los hijos varones. En la actualidad, los hogares transnacionales se configuran también con mujeres autónomas que han abandonado solas sus países, dejando en ellos a familiares dependientes por razones económicas y no por reagrupación familiar (reunirse ellas y sus hijos con el esposo). Este fenómeno ha cobrado especial relevancia en la literatura científica.

En relación a lo expuesto, no sorprende constatar como la transnacionalidad ha supuesto una transformación en la configuración de la feminidad y la masculinidad. Así, a la anterior diferenciación de los tipos de hogares transnacionales habría que incluir (Oso, 1998): (a) migración de mujeres solas con familias dependientes en el lugar de origen (casadas que mantienen el hogar trasnacional -esposo, hijos u otros familiares-, mujeres solteras con familiares a cargo -padre, hermanos, sobrinos-, y cabeza del hogar monoparental -solteras, separadas o divorciadas, y viudas-); y (b) migración familiar (pioneras de la migración, migración de la pareja, o mujer reagrupada por el esposo u otros familiares).

En la actualidad, la migración es una estrategia de subsistencia económica familiar diferenciada por su carácter cooperativo, su alto nivel de previsión y cálculo, y la toma meditada de decisiones sobre quién, cuándo y dónde emigrar. Todo ello influenciado por la demanda y las oportunidades del mercado de trabajo de los países de origen y destino, la edad de los integrantes de la familia, las responsabilidades asociadas a cada uno de ellos, y el género, determinando, incluso, si se emigra individualmente o en familia (Parella, 2007; Mora, 2008).

Según el Fondo de Población de las Naciones Unidas (UNFPA, 2006), se ha producido una variación notable en la composición del género de los flujos migratorios mundiales, de varones en los inicios del siglo xx a un equilibrio de género a comienzos del xxi. Los movimientos migratorios de las mujeres ya no solo son concebidos para la reagrupación familiar, sino que constituyen un eje más en la inmigración laboral. Son diversos los factores vinculados a la economía global que explican las corrientes migratorias femeninas y el desarrollo de hogares transnacionales.

Existe una relación clara entre el aumento de la población migrante y la globalización del comercio y su soporte: la entrada de las mujeres en trabajos de baja cualificación y precarios (servicios domésticos, de cuidado personal, y servicios sexuales). La inserción de la mujer como fuerza de trabajo en la denominada «manufactura global» se puede explicar por la percepción social sobre las habilidades y destrezas femeninas para la realización de tareas delicadas y porque son consideradas como mano de obra más dócil que los varones. Estos últimos suelen desempeñar trabajos más pesados, como pueden ser los vinculados a la agricultura y a la construcción (Mora, 2008). 
Sin embargo, las cadenas mundiales de cuidados y la reproducción de las desigualdades de género, etnia y clase, ponen en relieve algunas paradojas (Solè, 2007; Oso, 2008):

- Sustitución de unas mujeres por otras: las mujeres (del sur) dejan a sus hijos en el país de origen, a cargo de otras mujeres, para cuidar a los hijos de otras mujeres (del norte). Así, la mujer autóctona es sustituida por la mujer inmigrante, y ésta última por otras mujeres de su familia que quedan al cuidado de sus hijos en el país de origen.

- Desarrollo de la jerarquía marido/esposa/doméstica: el servicio doméstico libera a la clase media de las labores del hogar, pero refuerza las estructuras patriarcales; no se redefinen tareas y roles pues la presencia de una mujer que asume las tareas domésticas rezaga el esfuerzo por asumir las tareas del hogar por parte del esposo y los hijos.

- Otra forma de explotación de la mujer: el alejamiento de las mujeres migrantes de sus hijos, dejándoles a cargo de otras mujeres y renunciando a sus propias tareas para asumir las de otras, supone una forma más de explotación.

La relación entre migración y género se establece en dos vías estrechamente vinculadas (Rivas y Gonzálvez 2011): la migración influye en las relaciones y prácticas tradicionales de género que se dan en las unidades familiares, afianzando las desigualdades y los roles tradicionales o desafiándolos; el género influye en quién migra, en por qué y cómo se toma la decisión de hacerlo, en las formas en que la migración tiene impacto en los propios migrantes, en las áreas emisoras y receptoras, y en los procesos de fragmentación y de reconfiguración familiar tras la migración.

\subsection{Migración: efectos en el individuo y en la familia}

De forma individual, el inmigrante sufre el efecto de los problemas derivados del «síndrome de Ulises», que también es definido como el «síndrome del inmigrante con estrés crónico y múltiple», y cuyas consecuencias son (Aparicio, 2011): (a) sentimiento de soledad, ya que en muchas ocasiones el inmigrante se encuentra solo en el país receptor, sin familia o amigos que le apoyen; (b) sentimiento de fracaso, porque no encuentra trabajo en aquellos sectores laborales en los que ha sido formado, y cuando accede al mundo laboral suele desempeñar actividades marginales que en la mayoría de las ocasiones desconoce y le provocan gran frustración; (c) lucha por sobrevivir («síndrome del superviviente»), que se produce en algunos casos; (d) terror y miedo, pues en ocasiones pueden llegar a ser extorsionados por mafias y organizaciones que les presionan para obtener beneficios.

Además, se pueden identificar problemas puntuales de orden social, psicológico, familiar y educativo, que el inmigrante experimenta y que marcan su historia de vida y el significado que éste otorga a su proceso migratorio: (a) por la inadaptación medioambiental, de tipo climatológico y geográfico; (b) derivados de la descontextualización lingüística, social y cultural (costumbres y tradiciones diferentes); (c) alimenticios; (d) por el desconocimiento jurídico y laboral (en casos de escasa experiencia formativa previa, cultura laboral diferente e ignorancia de las leyes básicas del país de acogida); (e) generados por la restructuración psicosocial y sociocultural; (f) por la pérdida de identidad, pérdidas ambiguas, experiencias negativas vividas en sus países de procedencia, estereotipos existentes en el país de acogida, etc., que causan daño al proceso de integración de la población inmigrante (Aparicio, 2011). 
La inmigración desestabiliza a las familias y crea tensiones en su seno; de hecho, se puede documentar el considerable número de separaciones y divorcios durante el proceso migratorio y los efectos sobre las relaciones paterno filiales (Santos, 2008).

La mujer adquiere grandes cotas de autonomía cuando es ella quién emigra en primer lugar. En este caso, se convierte en la proveedora económica de la familia, mientras su marido permanece en el país de origen. Esto puede tornarse como una dificultad en la familia, ya que reta al rol tradicional del varón, cuestiona su función de sustentador, y le sitúa en el ámbito doméstico al frente del cuidado del hogar (Parella y Cavalcanti, 2006; Parella, 2007). Esta situación, incómoda para la mayoría de los hombres, es resuelta procurando emigrar tan pronto como la situación económica de la mujer en el país de destino lo permite, y las mujeres adquieren la obligación moral de «traerse al marido» (Pedone, 2006). En ocasiones (por ejemplo, ante infidelidades), los hijos pueden llegar al punto de negociar su posición frente al rumbo que toman las relaciones conyugales de sus padres (Pedone, 2008).

Cuando la pareja es emigrada por amor (con frecuencia elevada de mujeres), se deja en manos de la pareja de acogida la responsabilidad de la incorporación a la sociedad receptora. Las mujeres salen de su país para llevar a cabo un proyecto migratorio con el fin de formar pareja en el país de destino y crear su propia familia de procreación. El proyecto migratorio de estas mujeres es vivido como un proyecto de vida caracterizado por una incorporación dependiente al nuevo país, la negación de su condición de migrante y la idea del no retorno, por lo que mantienen prácticas transnacionales más débiles que otros colectivos de mujeres inmigradas, no usan cadenas migratorias para ayudarse en la migración ni buscan la participación en comunidades transnacionales. Sin embargo, las más preparadas y con mejor posición sí tienden a relacionarse con sus compatriotas (Bodoque, y Soronellas, 2010).

No todos los noviazgos terminan en boda. En algunos casos, las mujeres renuncian al matrimonio y se independizan de la pareja a través de la que llegaron al país; son mujeres que disponen de más habilidades, recursos y formación (superior) para considerar la separación de la pareja (Bodoque, y Soronellas, 2010).

En cuanto a la "paternidad/maternidad a distancia», ésta fractura a las familias, separando geográficamente a sus miembros. Todo ello conlleva importantes costos emocionales tanto para los padres como para los hijos que permanecen (dolor, ansiedad, sacrificios, presiones financieras, y dificultad para atender las necesidades de cuidado de los miembros dependientes) (Parrella, 2007), lo que impacta en mayor medida en los hijos que suelen ser menos conscientes de lo que ocurre, por lo menos al inicio del proyecto migratorio. La paternidad/maternidad a distancia supone nuevas formas de llevar a cabo el cuidado y la educación de los hijos.

Aunque la separación geográfica y social pueda generar beneficios relacionados con el aumento de posibilidades económicas en estas familias, se producen también consecuencias de carácter negativo que pueden percibirse en la sociedad receptora (Gregorio, 1998; Martín, 2007; Parella, 2007; Oso, 2008; Zapata, 2009; Rodríguez, 2014), tanto para las madres que han emigrado dejando a sus hijos en el país de origen, como para los hijos que se han quedado en el país de origen al emigrar sus padres.

En las familias cuyos padres funcionan como pareja sólida a pesar de la distancia se tiende a plantear y a desarrollar un proyecto familiar compartido de mejora y crecimiento. Este proyecto se vertebra mediante acciones planificadas, acuerdos específicos y claros, y plazos definidos, generando una comunicación frecuente y periódica con el miembro de la familia que emigró. De igual manera, se 
tiende a reconfigurar los roles y las funciones que éste realizaba, con el fin de paliar o evitar una posible desestructuración y desorganización interna. Se ciñen con mayor frecuencia a este modelo las familias en las que es el padre quien abandona su país. Se mantiene la división de roles de padre proveedor y madre cuidadora con funciones de soporte para procesar la ausencia. Los niños y adolescentes disponen de más elementos organizadores y recursos anticipatorios para afrontar la separación y enfrentar su futuro; sienten que tienen algún control sobre su porvenir, que cuentan con el soporte de sus padres para sus planes, y no hay una discontinuidad vital en sus procesos personales. Por el contrario, en las familias de padres separados es generalmente la madre la que migra, por lo que en este grupo se concentra el mayor número de niños y adolescentes que viven sin sus progenitores. En estas familias la ausencia de la madre es vivida como una experiencia altamente desestabilizadora, y por lo general, el impacto que sufren es mayor en tanto que su organización familiar está más limitada.

\subsection{Inclusión de las familias inmigrantes en España en el contexto escolar}

Los proyectos migratorios son, a menudo, proyectos familiares acordados en el interior del hogar, y afectan por tanto a toda la familia. No obstante, acostumbra a ser solo uno de sus miembros el que emigra o, cabe decir, el que inicia la emigración, porque a ese primer desplazamiento le siguen otros hasta acabar, en muchos casos, en una reagrupación familiar del cónyuge, de los hijos e incluso de los padres (abuelos) y de los hermanos (Bodoque y Soronellas, 2010).

La reunificación o reagrupación familiar es el derecho de los migrantes a mantener la unidad de su familia, pudiendo para ello reunir consigo a determinados parientes en el país al que se han desplazado. Reconstruir una familia a través de la reagrupación, mantenerla en la distancia desde el país de destino, o retomarla tras el retorno, puede definirse como una tarea ardua de años de esfuerzo y trabajo, repleta de obstáculos y contradicciones; de ahí las redefiniciones y cambios en las estrategias familiares en las sucesivas etapas del proyecto migratorio (Pedone, 2008).

Una vez reagrupada la familia en la sociedad o país de acogida, ésta pasa a considerarse familia inmigrante. Pero debido al contexto socioeconómico y a la complejidad de situaciones familiares, las reagrupaciones familiares no siempre son armoniosas; por el contrario, un número significativo de padres llevan a sus hijos a los lugares de destino sin las condiciones socioeconómicas adecuadas. El aumento de niños en el sistema educativo español muestra la aceleración en los procesos de reagrupación familiar, y permite, además, corroborar la marcada feminización del movimiento migratorio. Sin embargo, desde el momento que los proyectos migratorios contemplan la reagrupación familiar con vistas a un asentamiento definitivo, uno de los objetivos prioritarios para la familia es garantizar el acceso a los servicios sociales, sanitarios y educativos. En este sentido, que los hijos accedan a una calidad educativa mejor, junto a la obtención de recursos económicos, constituyen para las personas migrantes uno de los hitos más significativos de la migración (Pedone, 2006).

La familia es uno de los elementos más importantes a considerar en la inclusión de los inmigrantes, y su atención no goza de la misma facilidad que defender los derechos familiares de los ciudadanos de acogida debido a las políticas familiares y migratorias, y a la problemática de la exclusión. Las variantes de atención a esta realidad demandan una comprensión integradora de las experiencias concretas de las familias inmigrantes.

La educación se erige como vector de inclusión social de las familias inmigrantes. La educación en general, y los centros escolares y las entidades que apoyan a estos colectivos en particular, son los ins- 
trumentos fundamentales para la inclusión de las familias de origen inmigrante, puesto que los centros escolares son un ámbito privilegiado para identificar a los niños en situaciones de riesgo social y para intervenir en la superación de las desventajas debidas a su bagaje familiar (Gaviria, 2014). En estos contextos se resalta la necesaria colaboración y participación de la familia, como prueba la numerosa literatura publicada últimamente sobre ello.

Es cierto que en muchas ocasiones el tiempo que transcurre hasta la reagrupación familiar es largo, lo cual es importante para la integración social, especialmente de los más jóvenes. Cuando la separación ha sido prolongada la andadura familiar se reanuda con más problemas que si ha sido corta, y la reagrupación está marcada por los desencuentros. Los hijos deben enfrentarse a una nueva situación en un contexto desconocido, caracterizado por la insuficiente atención de los padres o la superación del sentimiento de abandono tras largas estancias (Santos, 2008). Por otro lado, los adolescentes que proceden de reagrupaciones conflictivas o de hogares desarticulados tienen que encontrar formas que les permitan continuar con sus procesos identitarios en un contexto social que les es adverso, y entonces aparecen reafirmadas las pertenencias culturales y territoriales (Pedone, 2006).

La participación de los padres en el centro escolar es uno de los factores que facilita, desde el contexto familiar, la integración escolar y social en la sociedad de acogida del alumnado inmigrante. En España, la mayoría de las familias inmigrantes que tienen relación con la escuela responden a un patrón mayoritario de matrimonio con hijos, y en menor medida de madres o padres solos con hijos. Diversas investigaciones sitúan la participación de los padres inmigrantes por debajo de los autóctonos, siendo mayor la participación en Educación Primaria Obligatoria que en Educación Secundaria Obligatoria; del mismo modo evidencian que cuanto mayor es el nivel de estudios de los padres mayor es el rendimiento escolar de los hijos, lo cual se observa con mayor cuantía en las familias inmigrantes (Santos, 2008).

La participación de las familias en la escuela puede verse obstaculizada por causas diversas: horarios laborales o características familiares (estatus de los miembros dentro del sistema familiar); desconocimiento de la cultura escolar del país de acogida; escaso reconocimiento de los bagajes culturales y las lenguas familiares; rechazo de las prácticas culturales y religiosas individuales y comunitarias; discrepancias en las formas de socialización de niños y jóvenes; proliferación de mensajes unidireccionales escuela-familia; existencia de códigos contradictorios; resistencias por parte de los grupos de familias autóctonas que manejan las asociaciones de madres y padres; características organizativas del propio sistema educativo (vías reales de participación) (Santos, 2008; Carrasco, Pàmies y Beltrán 2009). En este sentido, es importante llamar la atención sobre algo que se supone muy obvio a primera vista, pero que a veces parece necesario recordar. Los padres y las madres, al igual que sus hijos, no llegan vacíos de conocimientos y de experiencias escolares (Moscoso, 2009), y por lo tanto sería muy útil dentro del sistema educativo hacer acopio de estas experiencias para enriquecerlas y cimentar sobre ellas nuevos conocimientos académicos y del funcionamiento general de la escuela.

La escasa presencia en la escuela de las familias inmigrantes se sigue considerando un indicador de desinterés, e interpretando como un freno para el éxito de los hijos, desplazando la responsabilidad a la familia de que los hijos obtengan logros escolares, pues con frecuencia el profesorado infravalora el papel fundamental que desempeña la educación de los hijos en los proyectos migratorios familiares (Carrasco, Pàmies y Beltrán 2009). Encontramos algunos estudios, como el realizado por Meza, y 
Pederzini (2007), que contradicen esta percepción, dando cuenta del interés especial que muestran los padres inmigrantes en la educación de sus hijos, tanto si permanecen en el lugar de origen esperando la reagrupación familiar o si ya han podido dar este paso.

Por otra parte, los estudios realizados con población escolar inmigrante en España informan que los estudiantes inmigrantes experimentan situaciones de exclusión de diversa índole (Grau y Fernández, 2016). Así, los datos muestran que los alumnos inmigrantes tienen resultados escolares inferiores a los autóctonos en las puntuaciones del informe PISA 2012 (OCDE, 2013), y bajan considerablemente la puntuación global del país, aunque es diferente según la comunidad autónoma (Carabaña, 2008); las comunidades con mayor número de alumnado inmigrante han obtenido peores resultados y las comunidades con menor número, mejores resultados (Santos, Lorenzo y Priegue, 2011). Tal y como lo menciona Moscoso (2009), resulta por lo menos curioso que las percepciones de los padres no consideren la exclusión a la que sus hijos podrían verse sometidos por su origen; de hecho, al preguntar sobre este punto, la mayoría piensa que sus hijos e hijas reciben el mismo trato que sus compañeros nacionales.

Existe una gran presencia de alumnado inmigrante en Educación Primaria Obligatoria, con un abandono mayor que los autóctonos en la Educación Secundaria Obligatoria, y descendiendo considerablemente en los niveles no obligatorios (Educación Infantil, Bachillerato, Formación Profesional y Universidad) (Carabaña, 2008). Asimismo, en algunos grupos de emigrantes existe un alto índice de absentismo escolar y de alumnado por escolarizar (Arjona, Checa y Checa, 2014).

La transición al mundo del trabajo de los jóvenes inmigrantes respecto a los autóctonos se caracteriza por: vivir en mayor proporción exclusivamente de sus ingresos (un 38\% frente al 23\% de los autóctonos); entre los que tienen recursos propios predominan los trabajos esporádicos; los que no tienen ingresos propios suelen recibirlos de sus parejas más que de sus familias; y los ingresos netos son un $12 \%$ inferiores a los de los autóctonos. Tienen mayor autonomía económica y se incorporan antes al mundo del trabajo, siendo los latinoamericanos los que más pronto lo hacen (Riesco y Carrasco, 2008), por lo que se independizan antes de la familia. La incorporación al mercado de trabajo es el objetivo primordial de la apuesta migratoria y de sus expectativas, quedando muy desplazada la formación. Prolongar la educación más allá del periodo obligatorio supone un gran esfuerzo para las familias inmigrantes, y se percibe como un obstáculo para la realización del proyecto migratorio tanto familiar como propio. Por otra parte, los jóvenes inmigrantes planifican menos el futuro y viven en un presente continuo, en el que las posibilidades de elección son menores y su disponibilidad para el empleo es mayor; por lo que son más proclives a ocupar trabajos menos cualificados y con peores condiciones, y a perpetuarse en este tipo de trabajos en los que la movilidad laboral es mínima. Asimismo, la cualificación profesional de los inmigrantes en sus países de origen no suele ser reconocida en España (Carrasco y Riesco, 2008; Arjona, Checa y Checa, 2014).

La menor duración de los periodos de aprendizaje, el abandono escolar temprano, con fuertes componentes de sacrificio y responsabilidad, y los itinerarios formativos elegidos (profesiones manuales) son las causas de las características de la transición profesional de estos jóvenes (Riesco y Carrasco, 2008).

Se asume que la existencia de estrechas relaciones entre familias y escuela repercute positivamente en la integración social y educativa de los niños y los jóvenes, y que los resultados académicos de los estudiantes mejoran cuando la implicación de las familias en la escuela es mayor (Carrasco, Pàmies y 
Bertrán, 2009). Si los padres son importantes en la inclusión escolar y social de sus hijos, así como en su rendimiento académico, es necesario desarrollar y poner en marcha estrategias de trabajo y mecanismos de colaboración teniendo en cuenta las particularidades de estas familias.

\section{Conclusiones}

Las familias transnacionales se constituyen a partir de los procesos migratorios, y son aquellas cuyos miembros viven separados físicamente unos de otros, pero manteniendo vivos los vínculos familiares, lo que les permiten sentirse parte de una unidad familiar a pesar de la distancia. Por ello, resulta adecuado estudiar la integración de las personas migrantes atendiendo a los vínculos y conexiones que éstas mantienen con sus lugares de origen, porque tales factores intervienen en el proceso de inclusión al igual que otros rasgos personales, como por ejemplo el nivel de estudios, la composición familiar, o la participación en asociaciones -o entidades como la escuela- (González-Rábago, 2016).

Estas familias se forman cuando uno de los progenitores emigra, o los dos, o lo hace un hijo adulto. Sin embargo, la literatura revela que cada vez son más frecuentes los hogares transnacionales configurados por mujeres autónomas que han abandonado sus países, dejando en ellos a sus hijos con el esposo, así como aquellos en los que se ha producido la migración familiar completa.

En los últimos años, el discurso elaborado desde los ámbitos políticos, mediáticos y socioeducativos, coloca a la feminización del proceso migratorio como la causa principal del abandono de los hijos e hijas y de la desintegración familiar, estigmatizando el desempeño escolar y social de niños, niñas y adolescentes involucrados en estos contextos migratorios transnacionales (Pedone, 2008).

Aunque los trabajos sobre maternidad y paternidad trasnacional son escasos y se centran en los aspectos económicos de la separación entre padres e hijos, en este trabajo se ha querido poner el acento en el importante coste emocional que supone para ambos subsistemas la distancia geográfica que separa a madres y padres de hijas e hijos, separación que es acusada especialmente por los hijos por ser menos conscientes de lo que ocurre, y por la madre cuando ésta es quien primero emigra.

Los estudios revisados sobre experiencias concretas de las familias inmigrantes ponen de manifiesto el impacto de la migración en el sistema familiar, y argumentan que la educación es uno de los elementos clave para la inclusión de las familias de origen inmigrante en la sociedad de destino. Así, en la nueva economía globalizada deben promoverse imaginarios sociales y actitudes favorables tanto para la adopción de una formación académica y una capacitación laboral más a tono con lo internacional, como de una personalidad expresa mediante procesos de socialización y educación de niños y jóvenes para operar en el marco de esta nueva economía y, por ende, con estilos de vida más internacionales (Ojeda, 2009). Explica la autora que combinar estas dos fuentes de influencia sobre la población contribuirá a crear un ambiente social más tolerante y propicio a la adopción de estilos de vida más abiertos hacia lo transnacional, lo internacional y lo intercultural, que busca y necesita ser parte de la globalización frente a los nuevos retos que le impone el desarrollo. Las familias transnacionales y las familias inmigrantes contribuyen a ese cambio de mentalidad a través de las generaciones futuras respecto de lo que se piensa acerca de los que viven en «otro país»y, por consiguiente, de «este país».

Mantener en la distancia una familia, o reconstruirla a través de la reagrupación, constituye un fuerte desafío para todos sus miembros, especialmente para los más jóvenes ya que deben integrarse en dos sistemas al mismo tiempo, la sociedad y la escuela. 
En cuanto al sistema escolar, los inferiores resultados académicos del alumnado extranjero respecto al autóctono, el abandono escolar después de la enseñanza obligatoria, su menor presencia en los niveles de enseñanza postobligatoria (lo que puede dar lugar a un trabajo inestable y de baja cualificación), los problemas de interrelación en los centros escolares del alumnado inmigrante, y la escasa participación de sus padres en la escuela, dificultan la inclusión de la población inmigrante en la sociedad española (Grau y Fernández, 2016).

La escuela puede y debe por tanto contribuir desde todas sus estancias a ese cambio de mentalidad, posibilitando «encuentros interculturales reflexivos» y otro tipo de actividades, aprovechando la presencia de niñas/os, padres y madres de diferentes nacionalidades, lo que se convierte en un capital de alto valor dentro de la institución con miras a hacerlo extensivo a todo contexto social en el cual se encuentra inmerso cada uno de los centros.

También la universidad debe dirigir la investigación futura a explorar cómo el profesorado universitario está desmantelando las visiones de déficit de los maestros y trabajar directamente sobre aspectos complejos relacionados con la igualdad, particularmente con estudiantes inmigrantes y de otras minorías (Wassell, Kerrigan y Fernández-Hawrylak, 2017).

Siendo comprensivos con la decisión de emigrar, en lugar de rechazar la ausencia de vínculos o su relación diferente (mantenidos, reconstruidos o retomados), favoreceríamos la unidad del grupo a pesar de la existencia de criterios diferentes ante situaciones comunes, y contribuiríamos a construir una sociedad intercultural.

\section{Referencias bibliográficas}

Aparicio, Jesús Ma (2011): "La educación intercultural en la cooperación al desarrollo de América Latina" en Jesús Ma Aparicio Gervás (dir.): Interculturalidad, Educación y Plurilingüismo en América Latina. Madrid: Pirámide.

Arjona, Ángeles, Checa, Juan Carlos y Checa, Francisco (2014): "Inmigración y acceso a la universidad. Modelo interactivo de análisis”. Gazeta de Antropología, 30 (2), 1-20.

Bisquerra, Rafael (2009). Metodología de la investigación educativa (2a ed.). Madrid: La Muralla.

Bodoque, Yolanda y Soronellas, Montserrat (2010): "Parejas en el espacio transnacional: los proyectos de mujeres que emigran por motivos conyugales". Migraciones Internacionales, 5 (3), 143-174.

Carabaña, Julio (2008): “El impacto de la inmigración en el sistema educativo español”. ARI, 63, 1-11.

Carrasco, Silvia; Pàmies, Jordi y Bertrán, Marta (2009): "Familias inmigrantes y escuela: Desencuentros, estrategias y capital social”. Revista Complutense de Educación, 20 (1), 55-78.

Cerda, Julia (2014): “Las familias transnacionales”. Espacios Transnacionales, 2, 78-88.

Day, Robert A. (2005). Cómo escribir y publicar trabajos cientificos ( $3^{\mathrm{a}}$ ed.). Washington DC: Organización Panamericana de Salud.

Gaviria, José Luis (coord.) (2014). La participación de las familias en la educación escolar. Madrid: Ministerio de Educación, Cultura y Deporte.

González-Rábago, Yolanda (2016): "Determinantes de la vinculación trasnacional de los migrantes: La población colombiana en el País Vasco”. Migraciones Internacionales, 8 (4), 231-267. 
Gonzálvez, Herminia (2007): "Familias y hogares transnacionales: Una perspectiva de género". Puntos de Vista. Cuadernos del Observatorio de las Migraciones y de la Convivencia Intercultural de la Ciudad de Madrid, 11, Año III, 7-26.

Grau, Claudia y Fernández, María (2016): "La educación el alumnado inmigrante en España”. ArXius de Ciències Socials, 34, 141-146.

Gregorio, Carmen (1998). Inmigración femenina: su impacto en las relaciones de género. Madrid: Narcea.

Lorenzo, Mar; Santos, Miguel Ángel y Godás, Agustín (2012): “Inmigración y educación. ¿Influye el nivel educativo de los padres en el rendimiento académico de los hijos?”. Teoría de la Educación, 24 (2), 129-148.

Martín, Consuelo (2007): "Nuevas direcciones para estudios sobre familia y migraciones internacionales". Aldea Mundo. Revista sobre Fronteras e Integración, año 11, 22, 55-66.

Mejía, Mildred Zuleika y Arriaga, José Luis (2012): “Conformación de la familia transnacional y reorganización de la unidad doméstica”. Nueva Época, año, 2(1), 100-117.

Meza, Liliana y Pederzini, Carla (2007): "Migración intergeneracional y escolaridad como medios alternativos de movilidad social: el caso de México". Estudios Económicos, número extraordinario, 163-206.

Mora, Claudia (2008): “Globalización, Género y Migraciones”. POLIS, Revista de la Universidad Bolivariana de Chile, 7 (20), 285-279.

Moscoso, María Fernanda (2009): "Perspectivas de padres y madres ecuatorianos sobre el contrato pedagógico entre la institución escolar y la familia en un contexto migratorio". Papeles de Trabajo sobre Cultura, Educación y Desarrollo Humano, 5 (1), 1-40.

OCDE (2013). PISA 2012. "Programa para la Evaluación Internacional de los Alumnos. Informe Español”. Volumen I: Resultados y contextos. Madrid: Ministerio de Educación, Cultura y Deporte.

OIT (2006). Datos sobre migración laboral (en línea). http://www.ilo.org/public/libdoc/ ilo/2006/106B09_492_span.pdf, consultado 1 de octubre de 2014.

Oso, Laura (1998). La migración hacia España de mujeres jefas de hogar. Madrid: Instituto de la Mujer. Ministerio de Trabajo y Asuntos Sociales, Serie Estudios, nº52.

Oso, Laura (2008): “Migración, género y hogares transnacionales” en Joaquín García Roca y Joan Lacomba (eds.): La inmigración en la sociedad española. Una radiografía multidisciplinar. Barcelona: Bellaterra.

Ojeda, Norma (2009): "Reflexiones acerca de las familias transfronterizas y las familias transnacionales entre México y Estados Unidos”. Frontera Norte, 21 (2), 7-30.

Parella, Sònia (2007): "Los vínculos afectivos y de cuidado en las familias transnacionales. Migrantes Ecuatorianos y peruanos en España”. Migraciones Internacionales, 4(2), 151-188.

Parella, Sònia y Cavalcanti, Leonardo (2006): "Una aproximación cualitativa a las remesas de los inmigrantes peruanos y ecuatorianos en España y su impacto en los hogares transnacionales”. REIS, Revista Española de Investigaciones Sociológicas, 116, 241-257.

Pedone, Claudia (2006): De l'Equador a Catalunya: El paper de la familia i les xarxes migratòrie. Barcelona: Fundació Jaume Bofill. 
Pedone, Claudia (2008): “-Varones aventureros- vs. -Madres que abandonan-: reconstrucción de las relaciones familiares a partir de la migración ecuatoriana”. REMHU. Revista Interdisciplinar da Mobilidade Humana, año XVI, 30, 45-64.

Riesco, Alberto y Carrasco, Concepción (2008): "Escuela, consumo y mercado de trabajo: la producción de la "juventud" entre los jóvenes de origen inmigrante". Revista de Educación, 345, 183-203.

Rivas, Ana María y Gonzálvez, Herminia (2011): "El papel de las remesas económicas y sociales en las familias transnacionales colombianas”. Migraciones Internacionales, 6 (2) 75-99.

Rodríguez, Dan (2014): "En torno al parentesco transnacional: Contextualización y consideraciones teórico-metodológicas". AIBR. Revista de Antropología Iberoamericana, 9 (2), 183-210.

Rodríguez, David y Valldeoriola, Jordi (2009). Metodología de la investigación. Barcelona: UOC.

Santos, Miguel Ángel; Lorenzo, Mar y Priegue, Diana (2011): “Infancia de la inmigración y educación: la visión de las familias”. RIE. Revista de Investigación Educativa, 29 (1), 97-110.

Santos, Miguel Ángel (2008): "Las familias inmigrantes en la escuela. ¿Cómo avanzar en su integración desde la perspectiva socioeducativa?” en Joaquín García Roca y Joan Lacomba (eds.): La inmigración en la sociedad española. Una radiografía multidisciplinar. Barcelona: Bellaterra.

Solé, Carlota (Dir.) (2007). Los vínculos económicos y familiares transnacionales. Los inmigrantes ecuatorianos y peruanos en España. Bilbao: Fundación BBVA.

UNFPA (2016). Estado de la población mundial 2006. Hacia la esperanza: Las mujeres y la migración internacional. Fondo de Población de las Naciones Unidas (en línea). http://www.unfpa.org/webdav/site/global/shared/documents/publications/2006/sowp06-sp.pdf, consultado el 1 de octubre de 2014.

UNFPA. Informe del Secretario General en la 46a sesión del Comité sobre población y desarrollo del Fondo de Población de las Naciones Unidas. (E/CN.9/2013/1) (en línea). http://www.un.org/esa/ population/cpd/cpd2013/SGreport13February.v2_ changes.accepted.FP_advance $\% 20$ unedited $\% 20$ version_converted.pdf, consultado el 1 de octubre de 2014.

Wassell, Beth; Kerrigan, Mónica y Fernández-Hawrylak, María (2017): “Teacher Educators in a Changing Spain: Examining Beliefs About Diversity in Teacher Preparation”. Teaching \& Teacher Education, 69 (1), 223-233.

Zapata, Adriana (2009): "Familia trasnacional y remesas: padres y madres migrantes". Revista Latinoamericana de Ciencias Sociales, niñery juventud, 7 (2), 1749-1769.

\section{Notas biográficas}

María Fernández-Hawrylak es profesora titular de Universidad de la Universidad de Burgos. Dirige el Grupo de Investigación TFS-Terapia, Familia y Salud de la Universidad de Burgos. Licenciada en Filosofía y Ciencias de la Educación y Doctora por la Universidad de Salamanca. Terapeuta Familiar acreditada por la FEATF. Tiene tres quinquenios docentes. Sus ejes de investigación están centrados en la atención a la diversidad y las necesidades socio-educativas en enfermedades crónicas, minoritarias, discapacidades, y alumnado inmigrante. Ha realizado diversas publicaciones con índices de calidad, participado en diver- 
sos proyectos de investigación y realizado estancias de investigación. Tiene reconocidos dos Sexenios de Investigación.

Davinia Heras Sevilla es profesora contratada Doctor en el Área de Didáctica y Organización Escolar en la Universidad de Burgos. Licenciada en Pedagogía y Doctora por la Universidad de Burgos. Su investigación se centra en la educación sexual y la coeducación, contando con diversas publicaciones sobre estas temáticas. Cuenta con un tramo de investigación reconocido. En la actualidad es Coordinadora del Máster Universitario en Profesor de Educación Secundaria Obligatoria y Bachillerato, Formación Profesional y Enseñanzas de Idiomas, y forma parte del grupo Grupo de Investigación TFS-Terapia, Familia y Salud, coordinando la línea de investigación «Educación afectivo-sexual, emocional y de género». 